\title{
Condições sanitárias e os cuidados prestados na Guerra do Paraguai
}

\author{
Condiciones sanitarias y los Cuidados prestados \\ en la Guerra del Paraguay
}

\section{Sanitarian conditions and care in the Paraguayan war}

Pedro Ruiz B Nassar ${ }^{1}$, Fernando Rocha Porto ${ }^{2}$, Simone Aguiar ${ }^{3}$, Juliane Aguiar da Rocha ${ }^{4}$, Anna Paula Ataide ${ }^{1}$ Doutorando do Programa de Pós Graduação em Enfermagem e Biociências da Universidade Federal do Estado do Rio de Janeiro.; telefono: +55 21988401808

${ }^{2}$ Pós Doutor em Enfermagem; Escola de Enfermagem Alfredo Pinto/ Universidade Federal do Estado do Rio de Janeiro. End:

Rua Dr. Xavier Sigaud, 290 - Urca - Rio de Janeiro - RJ - 22.290-180. Correo electrônico: ramosporto@openlink.com.br;

3 Doutoranda do Programa de Pós Graduação em Enfermagem e Biociências da Universidade Federal do Estado do Rio de Janeiro. End: Rua Dr. Xavier Sigaud, 290 - Urca - Rio de Janeiro - RJ - 22.290-180. Correo electrônico: siraguiar@hotmail.com; 4 Mestranda em Enfermagem- Escola de Enfermagem Alfredo Pinto/ Universidade Federal do Estado do Rio de Janeiro. End.: Rua Dr. Xavier Sigaud, 290 - Urca - Rio de Janeiro - RJ - 22.290-180. Correo electrônico: juliane_ar@globo.com;

5 Graduanda em Enfermagem- Escola de Enfermagem Alfredo Pinto/Universidade Federal do Estado do Rio de Janeiro. End:

Rua Dr. Xavier Sigaud, 290 - Urca - Rio de Janeiro - RJ - 22.290-180. Correo electrônico: annapaulaataide@gmail.com

Cómo citar este artículo en edición digital: Nassar, P:R:B., Porto, F., Aguiar, S., Aguiar da Rocha, J. y Ataide, A.P. (2016). Condições sanitárias e os cuidados prestados na Guerra do Paraguai.. Cultura de los Cuidados (Edición digital),20(45). Disponible en: < http://dx.doi.org/10.14198/cuid.2016.45.05.>

Correspondencia: Pedro Ruiz B Nassar. Rua Dr. Xavier Sigaud, 290 - Urca - Rio de Janeiro - RJ - 22.290-180.

Correo electrónico: pedrornassar@gmail.com

Recibido: 11/011/2015; Aceptado: 12/04/2016

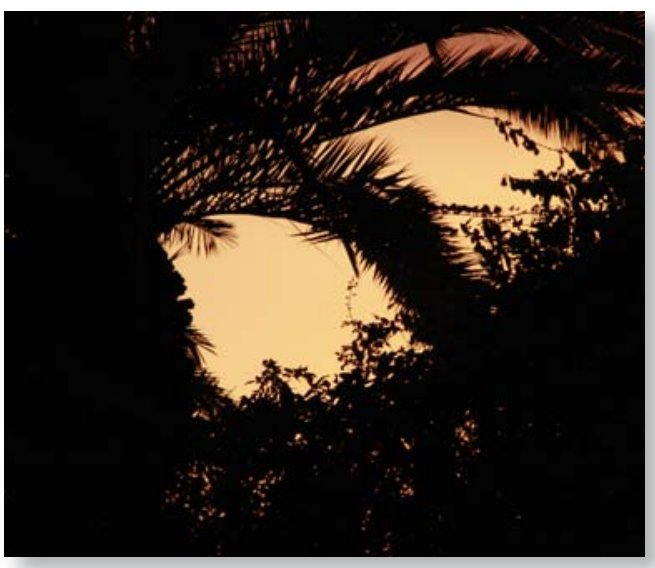

ABSTRACT

Objective: To analytically identify, in the periodical El Centinela, news related to the sanitary conditions and care towards the wounded in the Paraguay war.

Method: Serial history approach three news fragments were used to illustrate the sanitary conditions and care towards the wounded.
Results: The hostile sanitary conditions where the practice of care, compress with rips of skirts of women to stop the bleeding and the use of medicinal and nutritional marijuana were one of the methods used for care.

Conclusion: There wasn't the pretense of deepen the sanitary conditions and care to injured in action but carried that El Centinela periodical has regular daily richness of war, which allows somehow reassemble, even minimally, the history of care.

Keywords: War, Sanitary Conditions, Care and Nursing History.

\section{RESUMO}

Objetivo: Identificar, analiticamente, no periódico El Centinela, as notícias relacionadas com as condições sanitárias e os cuidados prestados aos feridos na guerra do Paraguai. 
Método: Estudo realizado com abordagem na história serial. Foram utilizados três fragmentos de notícias para ilustrar as condições sanitárias e os cuidados prestados aos feridos.

Resultados: As condições sanitárias hostis aonde ocorreram a prática do cuidado, bandagens com rasgos das saias das mulheres para estancar o sangue e o uso da coca medicinal e nutricional foram uma das formas utilizadas no cuidado.

Conclusão: Não houve a pretensão de aprofundar as condições sanitárias e os cuidados prestados aos feridos em combate, mas demostrou-se que o periódico El Centinela teve a riqueza diária da guerra, o que permite de alguma maneira, voltar a montar, mesmo que seja minimamente, a história do cuidado.

Palavras chave: Guerra, Perfis Sanitários, Cuidado e História da Enfermagem.

\section{RESUMEN}

Objetivo: Identificar, analíticamente, en el periódico El Centinela, las noticias relacionadas con las condiciones sanitarias y los cuidados prestados a los heridos en la guerra del Paraguay.

Método: Estudio en la abordaje de la historia serial. Fueron utilizados tres fragmentos de noticias para ilustrar las condiciones sanitarias y los cuidados prestados a los heridos.

Resultados: Las condiciones sanitarias hostiles dónde se ocurrió la práctica del cuidado, vendajes con rasgos de las faldas de las mujeres para estancar la sangre y el uso de la coca medicinal y nutricional fueron una de las formas se utilizadas en el cuidado.

Conclusión: No se ha pretendido profundizar en las condiciones sanitarias y los cuidados prestados a los heridos en combate, pero se demostró que periódico El Centinela tiene la riqueza diaria de la guerra, lo que permite de alguna manera, volver a montar, aunque sea mínimamente, la historia del cuidado.

Palabras chave: Guerra, Perfiles Sanitarios, Cuidado y Historia de la Enfermería.

\section{CONSIDERACIONES INICIALES}

El objeto del presente estudio son las noticias relacionadas a las condiciones sanitarias y los cuidados prestados a los heridos, por medio de la prensa paraguaya, en la Guerra de la Tríplice Alianza (1864-1870).

La Guerra de la Tríplice Alianza, comprendió el momento histórico donde a partir de las divergencias militares, políticas y geográficas, en la región del Plata, área que abarcaba la Bacía del Río del Plata (Toral, 2001), implicado: Uruguay, Argentina y Brasil contra Paraguay (Doratioto, 2002).

En 1864, el navío brasileño Marques de Olinda fue aprisionado en el Puerto de Asunción, hecho que potencializó negativamente las relaciones diplomáticas entre Brasil y $\mathrm{Pa}$ raguay, cuando en noviembre del mismo año el ejército liderado por Solano López invadió Mato Grosso (Brasil) y declaró guerra a la nación brasileña (Cerqueira, 2010).

Un año después de la invasión de tierras brasileñas, Solano López declaró guerra a Argentina, en lo que se refiere la negativa del pasaje por territorio argentino con la finalidad de invadir Uruguay y la región sur de Brasil. El intento de sorprender las tropas brasileñas en Uruguay, por medio del espacio de Argentina y sur de Brasil, ocurrió porque el Imperio Brasileño había apoyado a Venancio Flores para la presidencia de Uruguay articulado con Argentina, por motivos políticos y militares (Doratioto, 2002). El cuantitativo de involucrados en el conflicto de la Guerra de la Tríplice Alianza, por parte brasileña fue de, aproximadamente, 124 mil soldados, contando Argentina con 
cerca de 30 mil soldados y Uruguay con poco más de 5 mil hombres en su contingente. En Paraguay el resultado del conflicto fue trágico, teniendo en cuenta que la mayor parte se dio en territorio paraguayo y las condiciones de subsistencia eran escasas y las epidemias alcanzaron cerca de $70 \%$ de la población, tornándose la guerra más sangrienta de la América Latina (Doratioto, 2002).

Una de las estrategias del gobierno paraguayo para motivar su tropa en el conflicto, con la Tríplice Alianza, fue la utilización de la Prensa Nacional, por medio de los diarios El Semanario, EL Centinela, Cabichuí, Cacique Lambaré y La Estrella, en el sentido también de descalificar los adversarios. La prensa entendida como una de las formas del poder de hacer ver y creer, al vehicular noticia sobre el conflicto daba énfasis a las ventajas y conquistas del gobierno paraguayo, motivando sus tropas. Por otro lado, esos mismos ejemplares eran expresamente distribuidos en los campamentos de los aliados en el sentido de descalificarlos y hacer sobresalir a la soberanía de Paraguay. De esa manera, las noticias vehiculadas en los diarios, a la época, tenían el carácter informativo, de engrandecimiento de la nación y de las tropas paraguayas, con la intencionalidad de estimular la autoestima de los soldados, teniendo por efecto menospreciar la Tríplice Alianza. En este sentido, se tiene por objetivo identificar, analíticamente, en la prensa paraguaya las noticias relacionadas a las condiciones sanitarias y los cuidados prestados a los heridos en la guerra del Paraguay.

El presente estudio es producto de la construcción de una disertación de maestría, en curso, con la intención de identificar los cuidados prestados en la Guerra del Paraguay, teniendo en cuenta que diversos estudios en el campo de la historia describieron y analizaron en la óptica historiográfica, pero la propuesta aquí presentada trata de remontar la historia de los cuidados, en este caso, durante el conflicto ocurrido en la América del Sur.

Mediante al expuesto en los resultados de la investigación pueden colaborar para el entendimiento de las condiciones de higiene, como posibles factores de agravio a la salud, así como rellenar micro espacios existentes en la práctica del cuidado.

Además, la contribución del estudio para la enseñanza se justifica por el hecho del mismo posibilitar la descripción de prácticas históricas sobre el cuidado a los enfermos y heridos en el siglo XIX, en el contexto de la Guerra del Paraguay, lo que posibilitará mejor entendimiento sobre la trayectoria del desarrollo de las acciones, por ejemplo, cuando vamos de encuentro a ciertos tipos de herida inciso contusas, más precisamente por arma de fuego o corto contusas por arma blanca.

\section{MÉTODO Y FUENTES}

El método del presente estudio ocurrió en el abordaje de la historia serial. Este es entendido como una de las formas de organizarse en series o en unidades cronológicas, que pueda atribuir significación unívoca, relativamente, aplicada al objeto de los estudios en la historia social (Cardoso. \& Brignoli, 2002).

Hay que destacar que el abordaje serial fue utilizado en el sentido de la serialidad documental, pero por opción, no fueron utilizados los elementos constitutivos del método, como: variante, desvío padrón y otros, sino la proposición de la representación gráfica y de ella, la retirada de fragmentos noticiosos para ejemplificar las condiciones sanitarias y los cuidados prestados a los heridos en el conflicto.

La Prensa Nacional del Paraguay era responsable por los periódicos, a saber: EL Semanario impreso en lengua española, lengua 
oficial del Paraguay hasta los días de hoy, su circulación era semanal y sus noticias tenían cuño económico, mundial, asuntos generales y sobre la guerra; Cabichuí creado durante la guerra tenía como principal idioma el guaraní, lengua nativa paraguaya, su circulación era semanal, así como los periódicos, Cacique Lambaré y La Estrella, que hasta el momento se sabe que su lengua era el guaraní, no obstante no fueron encontrados relatos, imágenes y acervos, hasta el momento y; EL Centinela creado en 1867, más exactamente en 25/04/1867, fecha en que circuló su primer número. El último periódico mencionado arriba fue publicado e impreso los jueves, distribuidos en los campamentos y fortalezas paraguayas. Él era compuesto de 4 páginas - frente y respaldo - e impresas en 3 columnas por página. Además, él era redactado en el idioma español, lengua oficial de la República del Paraguay, que noticiaba hechos referentes al frente de batalla. Los criterios de selección para la utilización del periódico se dieron por medio de la disponibilidad del acervo de la Biblioteca Nacional de Rio de Janeiro, en idioma español y temática exclusiva sobre la guerra y de exclusión el uso del idioma guaraní.

Mediante aplicación de los criterios de inclusión e exclusión fue visto que el diario EL Centinela referente al número 1 a 36 , que correspondió al período de marzo a octubre del año de 1867, delimitación temporal del estudio. Para la cosecha de los datos fue confeccionado un instrumento de investigación compuesto de 6 campos a ser rellenado con las debidas informaciones: nombre del diario, fecha de la publicación y separadamente los registros por página. Por lo tanto, fue utilizado como criterio de busca de las noticias: condiciones sanitarias del ambiente y los cuidados prestados a los soldados en el conflicto.
Los resultados serán presentados por medio de un gráfico con la finalidad de identificar el cuantitativo de las publicaciones sobre condiciones sanitarias del ambiente y los cuidados prestados a los soldados en el conflicto, así como ejemplificar en tres facsímiles las noticias encontradas, como una de las posibilidades de remontarse, a través de elementos indiciarios, tal vez otra versión e interpretación del conflicto en aprecio, pero aquí con enfoque para la construcción de la historia del cuidado.

\section{RESULTADOS}

En los 36 ejemplares consultados fue posible identificar un total de 22 noticias referentes a las condiciones sanitarias y al cuidado, que fueron distribuidos en la frecuencia, por medio de la representación gráfica, en el intervalo de cuatro en cuatro publicaciones (figura n. 1).

\section{Figura $n^{\circ} 1$}

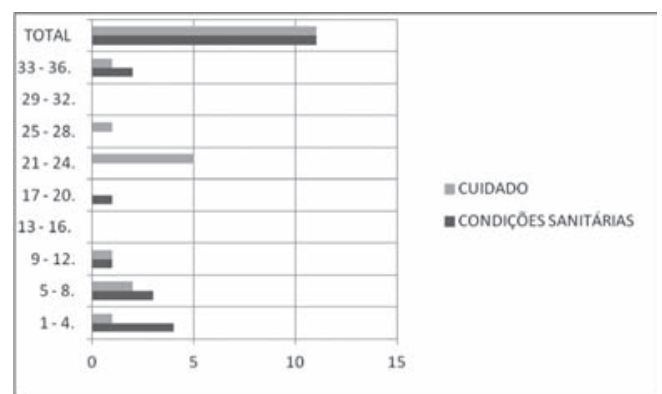

Fuente Diario "El Centinela" Noticias sobre la guerra del Paraguay (1867)

En el gráfico n.1, durante el año de 1867, fue identificada equidad en las noticias con abordaje de las condiciones sanitarias y del cuidado. Por otro lado, al observarse los intervalos en la representación gráfica, las noticias de cuidado surgen de forma más regular, cuando comparadas a las de las condiciones sanitarias.

Este resultado a simple vista posibilita inferir que el/los redactor(es) tal vez tuviese(n) 
más preocupación en divulgar materias sobre cómo se daba o qué era hecho frente a los acometidos de herimientos durante el frente en el teatro de guerra que las condiciones sanitarias. En esta perspectiva, las líneas que seguirán serán compuestas de un análisis de 1 noticia sobre las condiciones sanitarias y 2 con fragmentos de cuidados. Para comenzar, el primer facsímile se refiere a las condiciones sanitarias del ambiente, cabiendo el cuestionamiento, posiblemente a la época por lo lector: ¿Cuáles eran las condiciones sanitarias en el campo de batalla, considerando que en el período del conflito había carencia de alimentos, remedios, escasez de agua potable, entre tantos otros elementos, en el sentido de atenderse a las necesidades básicas humanas, sumado, todavía, a las condiciones climáticas?

\section{Facsímil no 1}

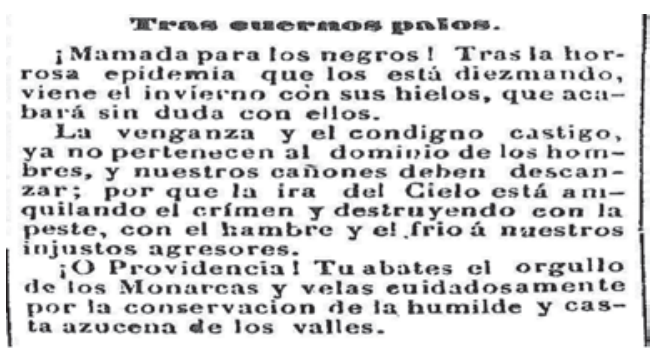

Fuente: El Centinela, 1867, $n^{\circ} 2, p g .3$

El facsímil 1 tiene por mensaje informar al lector, que el invierno era uno de los factores que podría acabar con los enemigos, entendidos por ellos (paraguayos) como los negros brasileños. Se entiende que, primeramente antes de realizarse el análisis del fragmento de la noticia, cabe considerar que las condiciones climáticas del período en Paraguay eran adversas de aquellas encontradas por los brasileños, en especial a aquellos de las regiones norte y nordeste, donde no estaban acostumbrados al verano caluroso, húmedo y con la presencia de las inundaciones de los ríos, causando constantes desbordamientos, diferentes del invierno riguroso a punto de surgir camadas de hielo por los campos al amanecer (Cerqueira, 2010). Cabe señalar que durante el conflicto, hubo varias víctimas relacionadas por las condiciones ambientales, como el frío, lo que favoreció la aparición de la fiebre catarral o la neumonía y la tuberculosis (Dourado, 2008).

Estas condiciones patológicas en conjunto con otras epidemias condujeron a la muerte millares de soldados de los países envueltos. El régimen político de Paraguay era republicano y de Brasil era imperialista, con adopción de la mano de obra esclava, que condicionaba al negro a trabajos forzados. Por este motivo ellos eran cedidos, por sus señores, como soldados voluntarios de la patria. En este sentido, ambos países tenían en su cuerpo militar esclavos negros, pero por situaciones adversas el negro brasileño era constantemente depreciado por los paraguayos, tal vez como una de las estrategias de demostrarse superiores en su manera de gobernar el país (Doratioto, 2002).

Además la crítica referente a la presencia de la etnia negra, el clima era un factor desfavorable, en el sentido que el Brasil tenía temperaturas más amenas cotejadas a la de los paraguayos, que se caracterizaba en la región geográfica de la guerra, presentaba un invierno con frío intenso, facto que diezmó casi un batallón intero oriundo de Pará. (Doratioto, 2002).

De esta forma, se puede deprender que, el clima en la región y temporalidad en cuestión, fue causador de dolencias y óbitos en virtud del ejército brasileño ser compuesto por personas oriundas del norte y nordeste del país y otras por ser de etnia negra. 


\section{Facsímil $n^{\circ} 2$}

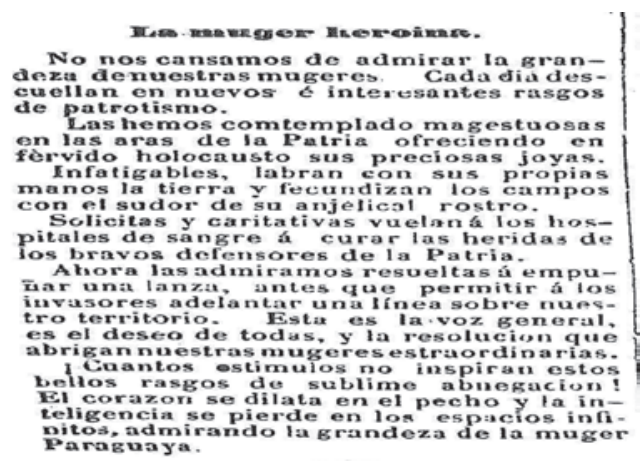

Fuente: El Centinela 1867, no 2 pg. 2

El fragmento del facsímile de número 2, titulado La mujer heroína, destaca la práctica del cuidado a los heridos de guerra, realizado por mujeres en un local denominado Hospital de sangre. Además de eso, aborda también que la mujer realizaba trabajos en el campo - agricultura - y mediante la necesidad participaba en el frente de batalla, en defensa de su patria.

Se destaca que Hospital de sangre, fue entendido como cualquier local adaptado para atender a los heridos de guerra, siendo él una institución nosocomial o no (Gómez, Lamelas, Rodriguez y Fernández, 1996/1997). El escenario de actuación para el cuidado era realizado por varias mujeres, de entre ellas, Jovita Feitosa, que al alistarse en Piauí, fue descubierta, aceptada y designada a un hospital militar (Doratioto, 2002). Se sabe que varias fueron las mujeres que participaron de la guerra, sea de forma directa o indirecta, pero, todavía, son parcos registros, incluso se piensa que muchas puedan ser desconocidas en la historiografía, pero las investigaciones en la historia vienen a lo largo del tiempo, evidenciando nombres femeninos. Sobre los cuidados prestados en el ambiente hospitalario, se sabe que, por ejemplo, el agua para los curativos venían en una vasija de hierro con gotas de ácido fénico, que en la época se creía que era capaz de interrum- pir la fermentación y la putrefacción orgánica, utilizadas en el lavado de las heridas y otras posibles conductas en la tentativa de evitarse amputaciones (Martins, Martins, Ferreira y Toledo, 1997; Cerqueira, 2010). Fuera del ambiente hospitalario, en especial en el frente de la guerra, para cuidarse de las heridas de los soldados, las mujeres rasgaban sus faldas para hacer vendajes y adaptar torniquetes y/o garrotes con la finalidad de estancar la sangre, en situación de hemorragia (Cerquira, 20109).

Se desprende que, a pesar del teatro de guerra no posibilitar un cuidado, posiblemente, más apropiado que si tuviese mejores condiciones materiales - ataduras, garrotes apropiados para contener las hemorragias, de entre otros - probablemente utilizados en hospitales de los centros urbanos, los cuidados prestados en aquellas condiciones sanitarias eran realizados mediante a los valores y concepciones aceptables en el sentido de salvarse la vida.

La próxima noticia se refiere al uso de la substancia de la coca, pero antes de analizarla, vale resaltar que con la difusión de la noticia y citaciones en este estudio, no se tuvo la intención de hacer apología o incentivo de prácticas curativas con el uso de la misma, sea de forma dicha y/o no dicha, sino analizar el cuidado, por medio de ella, utilizado como fines terapéuticos para el alivio del dolor, incluso delante de sus efectos colaterales.

\section{Facsímile n. 3}

1,as frupuslades de la coca, segun uns

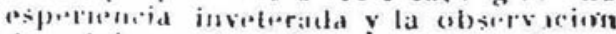
de mithicos afamadus, sull: eslinut mitog y linuder. fiura las indijesliunes de testomaso, lat diarreas y slrbe de alimento

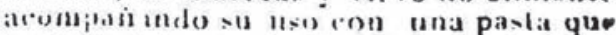

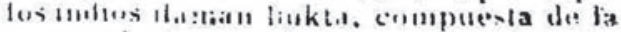

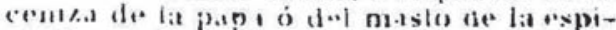
Ga d.1 mit. E-las minmas propiedaden

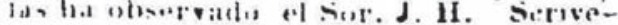

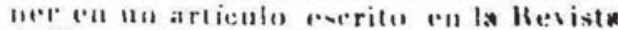
de Buenus Aire's de Novesubredel a ìn 68 .

Fuente: El Centinela 1867, no 23,pg. 1 
El fragmento del facsímile no 3 es referente a la noticia intitulada La coca. Esta destaca la práctica del cuidado en la atención a los acometidos con problemas gastrointestinales, curando las indigestiones del estómago y además, la hoja de coca, también era fuente de alimento juntamente con la maíz macerada, produciendo la crema de maíz.

Esa hoja, como se puede identificar, puede ser utilizada de dos maneras: medicinal y nutritiva. Sus propiedades son relatadas como uso continuo por los pueblos andinos, que utilizaban la hoja de coca como estimulante, para amenizar la fatiga provocada por los efectos de la altitud, para controlar las sensaciones de sed, hambre y soportar el frío intenso de las regiones altiplanas de la Cordillera de los Andes (Silva, 2007). Se destaca que el uso de la coca por los nativos de los Andes en América del Sur fue datado de 3.000 a.C.. Los indígenas de Perú, Bolivia y Colombia cultivan hace más de 1.000 años, teniendo el hábito de mascarlas o en el uso de tés, como forma de aliviar la fatiga y proporcionar bienestar (Cesena, 2011).

Se advertía, a la época, que el uso excesivo de esa hierba causaba daños, principalmente cuando en uso continuo, una vez que producía euforia, teniendo como efecto colateral la desnutrición en virtud de inhibir la sed y el hambre, dando sensación de plenitud y saciedad (Ferreira y Martini, 2013).

Se desprende que la hierba proporcionaba por un lado acción anestésica y bienestar, pero, por otro lado, la desnutrición, cuando en uso excesivo a los heridos en el conflicto. De esa manera, los cuidados eran prestados, por hombres y mujeres, pero el diario, para este momento, como opción de los investigadores el abordaje del registro obre la presencia femenina en la guerra.
Basado en tres fragmentos en la lengua española no se tiene la pretensión de asertivas en el sentido de generalizaciones, pero se puede por medio de ellas percibirse los vestigios e indicios dejados, que siendo organizados, en la lógica de la investigación, pueden rellenar huecos, sean ellas en que dimensión vayan para (des)cristalizar historias o cuentos contados, pero que académicamente merecerán su atención.

\section{CONCLUSIÓN}

El estudio presentó la equidad en las noticias sobre las condiciones sanitarias y los cuidados prestados a los heridos en combate, sin embargo la frecuencia de los registros noticiosos sobre el cuidado eran continuos. Esto significa que había, posiblemente, una intencionalidad en el sentido de propagarse el cuidado en los campamentos, en lo que se refiere las condiciones climáticas diferenciadas entre los países involucrados que, directa o indirectamente, influenciaban en las condiciones físicas del cuerpo militar.

A pesar de las condiciones climáticas en el campo de batalla ser distinta, para algunos miembros del cuerpo militar, en especial para los brasileños, estés enfrentaron, posiblemente, dos batallas, a saber: la primera, haber que enfrentar el frío de manera que el cuerpo no se encontraba preparado para soportar las condiciones inhóspitas y, la segunda, la guerra propiamente dicha.

Los cuidados prestados a los heridos en combate eran realizados, probablemente, en su mayoría por mujeres, por los relatos de otros investigadores citados en el presente estudio, siendo posible identificar que al mínimo hierbas y ungüentos eran utilizados para aliviar el sufrimiento y, que, la improvisación, por medio de paños, retales y otros utensilios 
eran utilizados para amenizar, o aun, resolver situaciones emergenciales que comprometían el umbral de la vida y muerte.

Así pues el estudio no tuvo la pretensión de evidenciar de forma microscópica y detallada las condiciones sanitarias y los cuidados prestados a los heridos en combate, a lo que se refiere la atención que se hace necesario la profundización en la temática.

Por otro lado, las noticias vehiculadas por el diario El Centinela conducen las riquezas del cotidiano de la guerra, pudiendo así inferirse como podría ser la práctica del día a día de la población que vivía en los Hospitales de sangre, campos de batalla y en sus alrededores, permitiendo, de cierta forma, remontarse, mismo que en pequeña parte, la historia del cuidado.

Esto significa que el diario El Centinela es una ventana que permite verse el pasado, en una otra perspectiva, tal vez, de algunas ya abordadas, lo que conduce a entender que la historia vista por la prensa se trata de un documento, todavía, poco explorado, pero de valor inestimable a los ojos de quien de él analiza e intenta producir conocimiento.

\section{BIBLIOGRAFÍA}

- Cardoso, C.F. \& Brignoli, H. (2002). Os métodos da história. $6^{\mathrm{a}}$ edição. Rio de Janeiro: Edições Graal.

- Cerqueira, D. (2010). Reminiscências da Campanha do Paraguai, 1865-70. Rio de Janeiro: Biblioteca do Exército.

- Cesena, F. (2011) Folhas de coca e cocaína: uma breve história de seus usos medicinais. Recuperado de: http:// www.cardiologiasemfronteiras.com.br/2011/10/folhas-de-coca-e-cocaina-uma-breve.html

- Doratioto, F. F. M (2002). Maldita guerra: Nova história da guerra do Paraguai. São Paulo: Cia. as Letras.

- Dourado, M. T. G. (2008) História das mulheres na guerra do Paraguai: fome e doenças sob a ótica do poder patriarcal. Universidade de São Paulo USP/FFLCH.
- Ferreira, P.E.M. y Martini, R.K. (2013). Cocaína: lendas, história e abuso. Rev. Bras. Psiquiatr. Recuperado de: http://www.scielo.br/scielo.php?script=sci arttext\&pid=S1516-4462001000200008\&lng=en.http:// dx.doi.org/10.1590/S151644462001000200008.

- Gómez, C.V.R., Lamelas,M.A.F., Rodriguez, R.A. \& Fernández, P.N (1996/97). La Enfermería en los Hospitales de Sangre. Galícia (1936-1939). Híades, Revista de historia de La Enfermería, 3 y 4 , 212-226.

- Martins, R.A; Martins, L.A.P., Ferreira, R.R. \& Toledo, C.F. (1997) Contagio: historia de la prevención de las dolienzas transmisibles. São Paulo: Moderna.

- Silva, F P . (2007). COCA - sagrada, medicinal e ilegal. Monografias Brasil Escola. Recuperado de: http://monografias.brasilescola.com/historia/cocasagrada-medicinal-ilegal.htm

- Toral, A. (2001). Imagens em desordem: a iconografia da Guerra do Paraguai. São Paulo: Humanitas / FFLCH /USP 